\title{
Fractional Factors, Component Factors and Isolated Vertex Conditions in Graphs
}

\author{
Mikio Kano* \\ Ibaraki University \\ Hitachi, Ibaraki, Japan \\ mikio.kano.math@vc.ibaraki.ac.jp \\ Hongliang $\mathrm{Lu}^{\dagger}$ \\ School of Mathematics and Statistics \\ Xi'an Jiaotong University \\ Xi'an, Shaanxi, China \\ luhongliang@mail.xjtu.edu.cn

\section{Qinglin $\mathrm{Yu}^{\ddagger \S}$} \\ School of Science \\ Xi'an Polytechnic University, Xi'an, Shaanxi, China \\ Department of Mathematics and Statistics \\ Thompson Rivers University, Kamloops, BC, Canada \\ yu@tru.ca
}

Submitted: Feb 6, 2019; Accepted: Oct 12, 2019; Published: Nov 22, 2019

(C) The authors. Released under the CC BY license (International 4.0).

\begin{abstract}
For a graph $G$, a fractional $[a, b]$-factor is a real valued function $h: E(G) \rightarrow[0,1]$ that satisfies $a \leqslant \sum_{e \in E_{G}(v)} h(e) \leqslant b$ for all $v \in V(G)$, where $a$ and $b$ are real numbers and $E_{G}(v)$ denotes the set of edges incident with $v$. In this paper, we prove that the condition $i s o(G-S) \leqslant\left(k+\frac{1}{2}\right)|S|$ is equivalent to the existence of fractional $\left[1, k+\frac{1}{2}\right]$-factors, where $i s o(G-S)$ denotes the number of isolated vertices in $G-S$. Using fractional factors as a tool, we construct component factors under the given isolated conditions. Namely, (i) a graph $G$ has a $\left\{P_{2}, C_{3}, P_{5}, \mathcal{T}(3)\right\}$-factor if and only if iso $(G-S) \leqslant \frac{3}{2}|S|$ for all $S \subset V(G)$; (ii) a graph $G$ has a $\left\{K_{1,1}, K_{1,2}, \ldots\right.$, $\left.K_{1, k}, \mathcal{T}(2 k+1)\right\}$-factor $(k \geqslant 2)$ if and only if $i s o(G-S) \leqslant\left(k+\frac{1}{2}\right)|S|$ for all $S \subset V(G)$,where $\mathcal{T}(3)$ and $\mathcal{T}(2 k+1)$ are two special families of trees.
\end{abstract}

Mathematics Subject Classifications: $05 \mathrm{C} 70$

*Supported by JSPS KAKENHI Grant Number 16K05248.

${ }^{\dagger}$ Supported by the National Natural Science Foundation of China under grant number 11871391 and Fundamental Research Funds for the Central Universities.

${ }^{\ddagger}$ Supported by the Discovery Grant of NSERC of Canada, and Shaanxi Hundred-Talents Program.

$\S$ The corresponding author 


\section{Introduction}

In this paper, we mainly consider finite simple graphs, which have neither loops nor multiple edges. A graph that may have multiple edges but has no loops is referred to as a multigraph. When defining notation and definitions, we often call a multigraph as a graph for convenience. Given a graph $G$, let $V(G)$ and $E(G)$ be its vertex set and edge set, respectively. The number of vertices of $G$ is called its order and is denoted by $|G|$. On the other hand, the number of edges in $G$ is called its size and is denoted by $\|G\|$.

For a set $X$, the cardinality of $X$ is denoted by $|X|$. For a vertex $v$ of a graph $G$, the degree of $v$ in $G$ is denoted by $\operatorname{deg}_{G}(v)$ and the set of edges of $G$ incident with $v$ is denoted by $E_{G}(v)$. For two vertices $x$ and $y$, an edge joining them is denoted by $x y$ or $y x$. We denote by $I s o(G)$ the set of isolated vertices of $G$, and by iso $(G)$ the number of isolated vertices in $G$. Thus iso $(G)=|I s o(G)|$. For two disjoint vertex sets $X$ and $Y$ of $G$, the set of edges of $G$ joining $X$ to $Y$ is written as $E_{G}(X, Y)$ and $e_{G}(X, Y):=\left|E_{G}(X, Y)\right| . P_{n}$ and $C_{n}$ are the path and the cycle of order $n$, respectively. The set of non-negative integers is denoted by $\mathbf{Z}^{*}=\{0\} \cup \mathbf{Z}^{+}$.

Let $G$ be a graph, and let $g, f: V(G) \rightarrow \mathbf{Z}^{*}$ be two integer-valued functions with $g \leqslant f$, that is, $0 \leqslant g(x) \leqslant f(x)$ for all $x \in V(G)$. Then a spanning subgraph $F$ of $G$ is called a $(g, f)$-factor of $G$ if $g(x) \leqslant \operatorname{deg}_{F}(x) \leqslant f(x)$ for all $x \in V(G)$. For real-valued functions $g, f: V(G) \rightarrow \mathbf{R}$ with $g \leqslant f$, a fractional $(g, f)$-factor is a function $h: E(G) \rightarrow[0,1]$ that satisfies the following condition:

$$
g(v) \leqslant \operatorname{deg}^{h}(v):=\sum_{e \in E_{G}(v)} h(e) \leqslant f(v) \text { for all } v \in V(G)
$$

where $\operatorname{deg}^{h}(v)$ is called the $h$-degree of $v$ and $h(e)$ is a real number between 0 and 1 inclusive. If the values of $h$ are 0 and 1 only, then a fractional $(g, f)$-factor becomes a $(g, f)$-factor. Many results on fractional factors of graphs can be found in [9].

To study fractional factors, Yang, Ma and Liu [8] introduced a parameter, isolated toughness of a graph $G$, denoted by $I(G)$, which is defined as

$$
I(G)=\min \left\{\frac{|S|}{i s o(G-S)}: S \subseteq V(G), i s o(G-S) \geqslant 2\right\}
$$

if $G$ is not complete; otherwise, $I(G)=\infty$. A graph $G$ is called isolated $t$-tough if $I(G) \geqslant t$, where $t \geqslant 0$ is a real number.

For a set $\mathcal{S}$ of connected graphs, a spanning subgraph $F$ of $G$ is called an $\mathcal{S}$-factor or a component factor if each component of $F$ is isomorphic to an element of $\mathcal{S}$ (see (1) of Figure 1). For a set $\mathbb{S}$ of positive integers, a spanning subgraph $F$ of $G$ is called an $\mathbb{S}$-factor of $G$ if $\operatorname{deg}_{F}(x) \in \mathbb{S}$ for all vertices $x$ of $G$. For an integer $k \geqslant 0$, the set of vertices of $G$ with degree $k$ is denoted by $V_{k}(G)$, namely, $V_{k}(G)=\left\{v \in V(G): \operatorname{deg}_{G}(v)=k\right\}$.

For a tree $T$, the set of leaves is denoted by Leaf $(T)$, that is, $V_{1}(T)$. An edge of $T$ incident with a leaf is called a pendant edge. In particular, the number of leaves of $T$ is equal to that of pendant edges of $T$. 


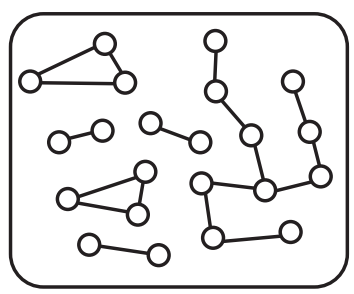

(1)

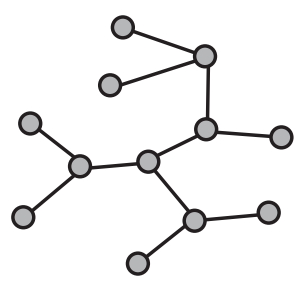

(2)

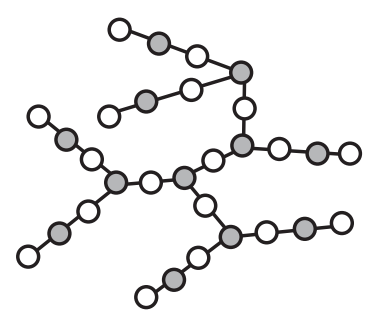

(3)

Figure 1: (1) A $\left\{P_{2}, C_{3}, P_{5}, \mathcal{T}(3)\right\}$-factor that contains no $P_{5}$-components; (2) A $\{1,3\}$-tree $R$; (3) The resulting $\{1,2,3\}$-tree $T_{R}$ obtained from $R$.

We define a special class of trees $\mathcal{T}(3)$ : for any $\{1,3\}$-tree $R$, which is a tree with vertex degrees 1 or 3 only, a new tree $T_{R}$ is obtained from $R$ by inserting a new vertex of degree 2 into every edge of $R$, and by adding a new pendant edge to every leaf of $R$ (see (2) and (3) of Figure 1 ). Then the tree $T_{R}$ is a $\{1,2,3\}$-tree having $\|R\|+\mid$ Leaf $(R) \mid$ vertices of degree 2 and has the same number of leaves as $R$. Also there is one-to-one correspondence between $V_{3}(R)$ and $V_{3}\left(T_{R}\right)$. The collection of such $\{1,2,3\}$-trees $T_{R}$ generated from all $\{1,3\}$-trees $R$ is denoted by $\mathcal{T}(3)$. A more general class of trees, $\mathcal{T}(2 k+1)(k \geqslant 2)$, will be defined in Section 2.

Tutte [7] established a relationship between isolated 1-tough graphs and $\left\{K_{2}, C_{n}: n \geqslant\right.$ $3\}$-factors.

Theorem 1 (Tutte [7]). A graph $G$ has a $\left\{K_{2}, C_{n}: n \geqslant 3\right\}$-factor if and only if

$$
\text { iso }(G-S) \leqslant|S| \text { for all } S \subset V(G) .
$$

Amahashi and Kano [2] extended Theorem 1 and gave a characterization for isolated $1 / k$-tough graphs in terms of star factors.

Theorem 2 (Amahashi and Kano [2]). Let $k \geqslant 2$ be an integer. A graph $G$ has a $\left\{K_{1, j}: 1 \leqslant j \leqslant k\right\}$-factor if and only if

$$
\text { iso }(G-S) \leqslant k|S| \text { for all } S \subset V(G) .
$$

Kano, Lu and $\mathrm{Yu}$ [5] obtained a sufficient condition for isolated 2-tough graphs to have component factors.

Theorem 3 (Kano, Lu and $\mathrm{Yu}[5])$. A graph $G$ has a $\left\{K_{1,2}, K_{1,3}, K_{5}\right\}$-factor if

$$
i s o(G-S) \leqslant \frac{|S|}{2} \quad \text { for all } \quad S \subset V(G)
$$

Kano and Saito [6] as well as Zhang, Yan and Kano [10] used isolated $k$-toughness to ensure the existence of special classes of component factors. 
Theorem 4 (Kano and Saito [6]). Let $k \geqslant 2$ be an integer. A graph $G$ has a $\left\{K_{1, j}: k \leqslant\right.$ $j \leqslant 2 k\}$-factor if

$$
\text { iso }(G-S) \leqslant \frac{|S|}{k} \quad \text { for all } S \subset V(G) .
$$

Theorem 5 (Zhang, Yan and Kano [10]). Let $k \geqslant 2$ be an integer. A graph $G$ has a $\left\{K_{1, j}, K_{2 k}: k \leqslant j \leqslant 2 k-1\right\}$-factor if

$$
i s o(G-S) \leqslant \frac{|S|}{k} \quad \text { for all } \quad S \subset V(G)
$$

In this paper, we carry on the investigations along the same direction mentioned above and obtain the factor characterizations of $I(G)=\frac{2}{3}$ and $I(G)=\frac{2}{2 k+1}(k \geqslant 2)$ :

Theorem 6. A graph $G$ has a $\left\{P_{2}, C_{3}, P_{5}, \mathcal{T}(3)\right\}$-factor if and only if

$$
\text { iso }(G-S) \leqslant \frac{3}{2}|S| \quad \text { for all } S \subset V(G) \text {. }
$$

Theorem 7. Let $k \geqslant 2$ be an integer. Then a graph $G$ has a $\left\{K_{1,1}, K_{1,2}, \ldots, K_{1, k}, \mathcal{T}(2 k+\right.$ 1) $\}$-factor if and only if

$$
i s o(G-S) \leqslant\left(k+\frac{1}{2}\right)|S| \text { for all } S \subset V(G) .
$$

\section{Proofs of Theorems 6 and 7}

For a function $f: V(G) \rightarrow \mathbf{Z}^{*}$ and a set $X$ of vertices of $G$, we write

$$
f(X):=\sum_{x \in X} f(x), \quad \text { in particular, } \quad \operatorname{deg}_{G}(X)=\sum_{x \in X} \operatorname{deg}_{G}(x) .
$$

The tools for proving Theorems 6 and 7 are fractional factors. We first describe the condition (3) in terms of fractional $\left[1, k+\frac{1}{2}\right]$-factors (Theorem 9), and then show that the minimal fractional factors are the desired component factors. In establishing the link between the condition (3) and fractional factors, we need the following result.

Theorem 8 (Anstee [3], Heinrich et al. [4]). Let $G$ be a multigraph and $g, f: V(G) \rightarrow \mathbf{Z}^{*}$ with $0 \leqslant g(x)<f(x)$ for all $x \in V(G)$. Then $G$ has a $(g, f)$-factor if and only if

$$
g(T)-\operatorname{deg}_{G-S}(T) \leqslant f(S) \quad \text { for all } S \subset V(G),
$$

where $T=\left\{v \in V(G)-S: \operatorname{deg}_{G-S}(v)<g(v)\right\}$.

If two functions $g, f: V(G) \rightarrow \mathbf{R}$ take constant real values $g(x)=a$ and $f(x)=b$ for every vertex $x$, then fractional $(g, f)$-factors are called fractional $[a, b]$-factors. 
Theorem 9. Let $k \geqslant 1$ be an integer and let $G$ be a graph. Then $G$ has a fractional $\left[1, k+\frac{1}{2}\right]$-factor $h$ with values in $\left\{0, \frac{1}{2}, 1\right\}$ if and only if

$$
\text { iso }(G-S) \leqslant\left(k+\frac{1}{2}\right)|S| \quad \text { for all } S \subset V(G) .
$$

Proof. Assume that $G$ satisfies (5). Let $G^{*}$ denote the multigraph obtained from $G$ by replacing each edge $e$ of $G$ by two parallel edges $e(1)$ and $e(2)$. Then $V\left(G^{*}\right)=V(G)$, and $\operatorname{deg}_{G^{*}}(v)=2 \operatorname{deg}_{G}(v)$ for every $v \in V\left(G^{*}\right)$. Define two functions $g, f: V\left(G^{*}\right) \rightarrow \mathbf{Z}^{*}$ as

$$
g(x)=2 \quad \text { and } \quad f(x)=2 k+1 \quad \text { for all } \quad x \in V\left(G^{*}\right) .
$$

Then $g<f$, and for any $S \subset V\left(G^{*}\right)$, we have

$$
\begin{aligned}
T & =\left\{v \in V\left(G^{*}\right)-S: \operatorname{deg}_{G^{*}-S}(v)<g(v)=2\right\} \\
& =\left\{v \in V\left(G^{*}\right)-S: \operatorname{deg}_{G^{*}-S}(v)=0\right\} . \\
& =I s o(G-S) .
\end{aligned}
$$

Thus it follows from the above equality and (5) that

$$
\begin{aligned}
g(T)-\operatorname{deg}_{G^{*}-S}(T) & =2 \cdot i \operatorname{so}(G-S)-0 \\
& \leqslant 2 \cdot\left(k+\frac{1}{2}\right)|S|=(2 k+1)|S|=f(S) .
\end{aligned}
$$

Hence by Theorem $8, G^{*}$ has a $(g, f)$-factor $F$. Now we construct a fractional $\left[1, k+\frac{1}{2}\right]$ factor $h: E(G) \rightarrow\left\{0, \frac{1}{2}, 1\right\}$ as follows: for an edge $e$ of $G$, (i) if $F$ contains both edges $e(1)$ and $e(2)$ of $G^{*}$, then define $h(e)=1$; (ii) if $F$ contains exactly one of $e(1)$ and $e(2)$, then define $h(e)=\frac{1}{2}$; (iii) otherwise, let $h(e)=0$. It is easy to see that $h$ is the desired fractional $\left[1, k+\frac{1}{2}\right]$-factor with values in $\left\{0, \frac{1}{2}, 1\right\}$.

Next assume that $G$ has a fractional $\left[1, k+\frac{1}{2}\right]$-factor $h$ with values in $\left\{0, \frac{1}{2}, 1\right\}$. Let $S \subset V(G)$, and let $F$ be the spanning subgraph of $G$ induced by $\{e \in E(G): h(e) \in$ $\left\{\frac{1}{2}, 1\right\}$. Clearly, the neighbors of each isolated vertex $u$ of $G-S$ are contained in $S$ and $\operatorname{deg}^{h}(u) \geqslant 1$, thus we have

$$
\begin{aligned}
i s o(G-S) & \leqslant \sum_{e \in E_{F}(\operatorname{Iso}(G-S), S)} h(e) \\
& \leqslant \sum_{x \in S} \operatorname{deg}^{h}(x) \leqslant\left(k+\frac{1}{2}\right)|S| .
\end{aligned}
$$

Hence $i s o(G-S) \leqslant\left(k+\frac{1}{2}\right)|S|$, or (5) holds.

Proof of Theorem 6. We first show that every tree $T \in \mathcal{T}(3)$ satisfies the condition (2). Define a function $h: E(T) \rightarrow\left\{\frac{1}{2}, 1\right\}$ as follows: for every pendant edge $e_{1}$ of $T$, let $h\left(e_{1}\right)=1$ and for any other edge $e_{2}$, let $h\left(e_{2}\right)=\frac{1}{2}$. Since $T$ is a $\{1,2,3\}$-tree and no 
pendant edge is incident with a vertex of degree $3, h$ is a fractional $\left[1, \frac{3}{2}\right]$-factor. Hence, by Theorem $9, T$ satisfies the condition (2).

Assume that $G$ has a $\left\{P_{2}, C_{3}, P_{5}, \mathcal{T}(3)\right\}$-factor $F$. Let $D_{1}, D_{2}, \ldots, D_{m}$ be the components of $F$. Then each $D_{i}$ is $P_{2}, C_{3}, P_{5}$ or a tree in $\mathcal{T}(3)$, and thus iso $\left(D_{i}-X_{i}\right) \leqslant \frac{3}{2}\left|X_{i}\right|$ for every $X_{i} \subset V\left(D_{i}\right)$. Then for any $S \subset V(G)$, we have

$$
\begin{aligned}
i s o(G-S) & \leqslant i s o(F-S)=\sum_{i=1}^{m} i s o\left(D_{i}-\left(S \cap V\left(D_{i}\right)\right)\right) \\
& \leqslant \sum_{i=1}^{m} \frac{3}{2}\left|S \cap V\left(D_{i}\right)\right|=\frac{3}{2}|S| .
\end{aligned}
$$

Hence the necessity is proved.

Next we prove the sufficiency. By Theorem 9, $G$ has a fractional $\left[1, \frac{3}{2}\right]$-factor $h$ with values in $\left\{0, \frac{1}{2}, 1\right\}$. We call an edge $e_{1}$ with $h\left(e_{1}\right)=1$ a red edge and an edge $e_{2}$ with $h\left(e_{2}\right)=\frac{1}{2}$ a blue edge. Let $F$ be the subgraph of $G$ induced by the set of all red and blue edges. Namely, $F$ is obtained from $G$ by removing all the edges $e_{3}$ with $h\left(e_{3}\right)=0$. Since $h$ is a fractional $\left[1, \frac{3}{2}\right]$-factor, $F$ is a spanning subgraph of $G$, and for every vertex $v$ of $G$, one of the following two statements holds:

(i) no red edge is incident with $v$ and two or three blue edges are incident with $v$; or

(ii) exactly one red edge is incident with $v$ and at most one blue edge is incident with $v$.

Choose a fractional $\left[1, \frac{3}{2}\right]$-factor $h$ so that the number of edges in $F$ is as small as possible. For the convenience, we also call $F$ a fractional $\left[1, \frac{3}{2}\right]$-factor. It is clear that $\operatorname{deg}_{F}(v) \in$ $\{1,2,3\}$. On the other hand, the degree $\operatorname{deg}^{h}(v)$ of $v$ in $h$ is 1 or $\frac{3}{2}$.

Claim 1. Every cycle of $F$ is a $C_{3}$-component in $F$.

Proof. Assume that $F$ contains a cycle $C$. First assume that $C$ is of even order. Take a perfect matching $M$ of $C$, and recolor all the edges of $M$ red, and remove all the edges in $C-M$. Then the resulting subgraph is a new fractional $\left[1, \frac{3}{2}\right]$-factor with red and blue edges, but its size is a smaller than $F$, which contradicts the choice of $F$. Hence $C$ is of odd order.

Assume that $C$ has two adjacent vertices $u_{1}$ and $u_{2}$ with degree 3 in $F$. Then $F-u_{1} u_{2}$ is a new fractional $\left[1, \frac{3}{2}\right]$-factor with fewer edges than $F$, which contradicts the choice of $F$. Hence if a vertex $v$ of $C$ has degree 3 in $F$, then the two neighbors of $v$ in $C$ have degree 2 in $F$. Assume that $C$ has a vertex $v$ with $\operatorname{deg}_{F}(v)=3$. Let $u_{1}$ and $u_{2}$ be the two neighbors of $v$ in $C$. Take a perfect matching $M$ of $C-v$. Recolor the edges of $M$ red, and remove all the edges of $(C-v)-M$ and $v u_{2}$. Since $v u_{1}$ and $v u_{2}$ are both blue edges, we obtain a new fractional $\left[1, \frac{3}{2}\right]$-factor with fewer edges than $F$, which is a contradiction. Hence $C$ is a component in $F$.

Moreover, it is easy to see that an odd cycle of $C$ order at least 5 has a $\left\{P_{2}, P_{5}\right\}$-factor $F_{C}$. Remove all the edges of $C$ not contained in $F_{C}$, recolor the edges contained in $P_{2}$ of $F_{C}$ red, and two pendant edges of $P_{5}$ of $F_{C}$ red and the remaining two edges of $P_{5}$ of $F_{C}$ blue. 
Then we obtain a new fractional $\left[1, \frac{3}{2}\right]$-factor with fewer edges than $F$, a contradiction. Therefore every cycle contained in $F$ is $C_{3}$. Consequently Claim 1 is proved.

For the simplicity of statements, in the rest of the proof, we will use "a smaller fractional $\left[1, \frac{3}{2}\right]$-factor" to substitute the phase "a new fractional $\left[1, \frac{3}{2}\right]$-factor with fewer edges than $F^{\prime \prime}$.

Claim 2. Every non-cycle component of $F$ is $P_{2}, P_{5}$ or a tree of $\mathcal{T}(3)$.

Proof. Let $x$ and $y$ be two vertices of degree 3 in $F$ such that they are adjacent or connected by a path whose inner vertices all have degree 2 in $F$. If $x$ and $y$ are adjacent in $F$, then $F-x y$ is a smaller fractional $\left[1, \frac{3}{2}\right]$-factor, a contradiction. Assume that $x$ and $y$ are connected by a path $\left(x, u_{1}, u_{2}, \ldots, u_{n}, y\right)$ of length at least $3(n \geqslant 2)$ such that every $u_{i}$ has degree 2 in $F$. We remove $u_{n} y$, recolor $u_{n-1} u_{n}$ red, and recolor all remaining edges of the path blue. Then the resulting subgraph is a smaller fractional $\left[1, \frac{3}{2}\right]$-factor, a contradiction. Therefore,

(a) if two vertices of $F$ with degree 3 are connected by a path in $F$ whose inner vertices all have degree 2 in $F$, then the length of the path is 2.

Let $z$ be a leaf of $F$ and let $x$ be a vertex of degree 3 in $F$. If $z$ and $x$ are adjacent,

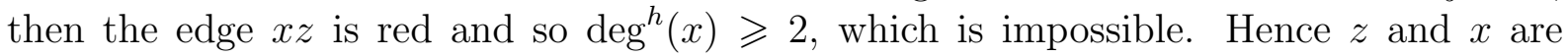
not adjacent. Assume that $z$ and $x$ are connected by a path $\left(z, u_{1}, u_{2}, \ldots, u_{n}, x\right)$ with $\operatorname{deg}_{F}\left(u_{i}\right)=2$ for every $i$. Then $z u_{1}$ is red. First assume that $n \geqslant 3$. Then remove $u_{n} x$, recolor $u_{n-1} u_{n}$ red, and recolor all the remaining edges of the path except $z u_{1}$ blue. Then the resulting subgraph is a smaller fractional $\left[1, \frac{3}{2}\right]$-factor, a contradiction. Next assume $n=1$. Then by removing $u_{1} x$, we obtain a smaller fractional $\left[1, \frac{3}{2}\right]$-factor, a contradiction again. Therefore,

(b) if a leaf $z$ is contained in a component $D$ of $F$ with $\Delta(D) \geqslant 3$, then there is a path $\left(z, u_{1}, u_{2}, x\right)$ in $F$ such that $\operatorname{deg}_{F}\left(u_{i}\right)=2$ and $\operatorname{deg}_{F}(x)=3$.

Consequently, if a component $D$ of $F$ contains at least two vertices of degree 3 , then by (a) and (b), $D$ is a tree of $\mathcal{T}(3)$. If $D$ has exactly one vertex of degree 3 , then by (b), $D$ is also a tree of $\mathcal{T}(3)$. If $D$ has no vertex of degree 3 , then $D$ is a path. It is obvious that $P_{3}$ has no fractional $\left[1, \frac{3}{2}\right]$-factors, and so $D$ is not $P_{3}$. If $D$ is a path of even order, then $D$ has a $P_{2}$-factor, and so it contradicts the minimality of $F$. If $D$ is a path $P_{n}$ of odd order with $n \geqslant 7$, then $D$ has a $\left\{P_{2}, P_{5}\right\}$-factor and thus contradicts the minimality of $F$. Hence Claim 2 holds.

Clearly, Claims 1 and 2 imply the sufficiency.

To state Theorem 7 , we need a new class of trees $\mathcal{T}(2 k+1)$. Let $k \geqslant 2$ be an integer and let $R$ be a tree that satisfies the following conditions: for every vertex $v \in V(R)$,

(i) $\operatorname{deg}_{R-\operatorname{Leaf}(R)}(v) \in\{1,3, \ldots, 2 k+1\}$, and

(ii) $2 \cdot($ the number of leaves adjacent to $v$ in $R)+\operatorname{deg}_{R-\operatorname{Leaf}(R)}(v) \leqslant 2 k+1$ in $R$. 


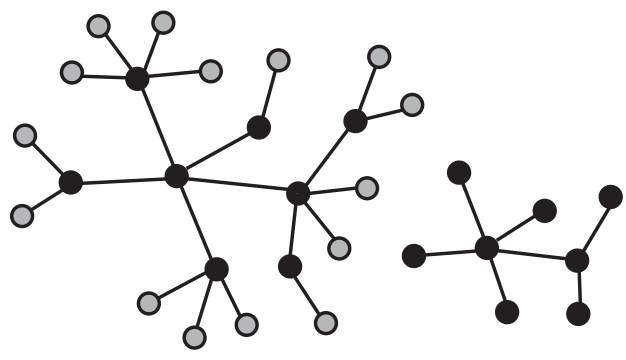

(1) $R$

(2) $R$ - Leaf(R)

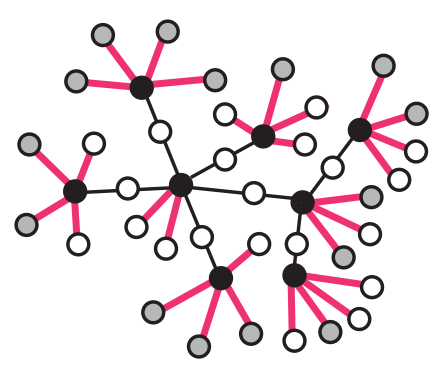

(3) $T_{R}$

Figure 2: (1) A tree $R$ that satisfies (6) with $k=4$; (2) The tree $R$ - Leaf $(R)$; (3) The tree $T_{R}$ obtained from $R$, where all red edges $e_{1}$ have value $h\left(e_{1}\right)=1$ and all other edges $e_{2}$ have value $h\left(e_{2}\right)=\frac{1}{2}$.

For such a tree $R$, we obtain a new tree $T_{R}$ as follows:

(iii) insert a new vertex of degree 2 into each edge of $R-\operatorname{Lea} f(R)$, and

(iv) for each vertex $v$ of $R-\operatorname{Leaf}(R)$ with $\operatorname{deg}_{R-\operatorname{Leaf}(R)}(v)=2 r+1<2 k+1$ in $R$, add $k-r-($ the number of leaves adjacent to $v$ in $R$ ) pendant edges to $v$ (see (3) of Figure 2 ).

Then the resulting tree $T_{R}$ has a fractional $\left[1, k+\frac{1}{2}\right]$-factor $h$ such that every pendant edge $e_{1}$ of $T_{R}$ has $h\left(e_{1}\right)=1$, all other edges $e_{2}$ have $h\left(e_{2}\right)=\frac{1}{2}$. Moreover, this fractional $\left[1, k+\frac{1}{2}\right]$-factor $h$ satisfies that every vertex $v$ of $R-\operatorname{Leaf}(R) \operatorname{has} \operatorname{deg}^{h}(v)=k+\frac{1}{2}$, and every leaf $x$ of $T_{R}$ and every inserted vertex $y$ of degree 2 have $\operatorname{deg}^{h}(x)=\operatorname{deg}^{h}(y)=1$ (see (3) of Figure 2). The set of such trees $T_{R}$ for all trees $R$ satisfying (6) is denoted by $\mathcal{T}(2 k+1)$. Note that the construction of $\mathcal{T}(3)$ and that of $\mathcal{T}(2 k+1)$ with $k \geqslant 2$ are similar, but adding pendant edges to some vertices of $R-\operatorname{Lea} f(R)$ is not defined in the construction of $\mathcal{T}(3)$.

Proof of Theorem 7. For any tree $T \in \mathcal{T}(2 k+1)$, since $T$ has a fractional $\left[1, k+\frac{1}{2}\right]$-factor $h$ of values $\left\{\frac{1}{2}, 1\right\}, T$ satisfies (3) by Theorem 9 .

Assume that $G$ has a $\left\{K_{1,1}, K_{1,2}, \ldots, K_{1, k}, \mathcal{T}(2 k+1)\right\}$-factor $F$. Let $D_{1}, D_{2}, \ldots, D_{m}$ be the components of $F$. Then each $D_{i}$ is $K_{1, s}$ for some $1 \leqslant s \leqslant k$, or a tree in $\mathcal{T}(2 k+1)$. Thus iso $\left(D_{i}-X_{i}\right) \leqslant\left(k+\frac{1}{2}\right)\left|X_{i}\right|$ for every $X_{i} \subset V\left(D_{i}\right)$. Then, for $S \subset V(G)$, we have

$$
\begin{aligned}
i s o(G-S) & \leqslant i s o(F-S)=\sum_{i=1}^{m} i s o\left(D_{i}-\left(S \cap V\left(D_{i}\right)\right)\right) \\
& \leqslant \sum_{i=1}^{m}\left(k+\frac{1}{2}\right)\left|S \cap V\left(D_{i}\right)\right|=\left(k+\frac{1}{2}\right)|S| .
\end{aligned}
$$

Hence the necessity is proved.

Next we prove the sufficiency. Assume that $G$ satisfies (3). By Theorem 9, $G$ has a fractional $\left[1, k+\frac{1}{2}\right]$-factor $h$ with values in $\left\{0, \frac{1}{2}, 1\right\}$. We call an edge $e_{1}$ with $h\left(e_{1}\right)=1$ a 
red edge and an edge $e_{2}$ with $h\left(e_{2}\right)=\frac{1}{2}$ a blue edge. Let $F$ be the subgraph of $G$ induced by the set of all red and blue edges. Namely, $F$ is obtained from $G$ by removing all the edges $e_{3}$ with $h\left(e_{3}\right)=0$, and since $h$ is a fractional $\left[1, k+\frac{1}{2}\right]$-factor, $F$ is a spanning subgraph. Choose a fractional $\left[1, k+\frac{1}{2}\right]$-factor $h$ of $G$ so that the number of edges in $F$ is as small as possible. It is clear that $1 \leqslant \operatorname{deg}_{F}(v) \leqslant 2 k+1$. On the other hand, the $h$-degree of $v$ can be expressed as $\operatorname{deg}^{h}(v)=1+\frac{1}{2} t$ for some integer $t, 0 \leqslant t \leqslant 2 k-1$.

Claim 1. F contains no cycles, and thus $F$ is a forest.

Proof. Suppose that $F$ contains a cycle $C$. First assume that $C$ is of even order. Take a perfect matching $M$ of $C$, and recolor all edges of $M$ red, and remove all edges in $C-M$. Then the resulting subgraph is a new fractional $\left[1, k+\frac{1}{2}\right]$-factor of $G$, but its size is a smaller than $F$, a contradiction. Hence $C$ is of odd order.

As in the proof of Theorem 6 , we substitute "a new fractional $\left[1, k+\frac{1}{2}\right]$-factor with less edges than $F$ " by "a smaller fractional factor" for the simplicity in the rest of the proof.

Assume that $C$ has two adjacent vertices $v_{1}$ and $v_{2}$ with degrees at least 3 in $F$. Then $F-v_{1} v_{2}$ is a smaller fractional factor, a contradiction. Hence if a vertex $v$ of $C$ has degree at least 3 in $F$, then the two neighbors of $v$ in $C$ have degree 2 in $F$. Assume that $C$ has a vertex $v$ with $\operatorname{deg}_{F}(v) \geqslant 3$. Let $u_{1}$ and $u_{2}$ be the two neighbors of $v$ in $C$. Take a perfect matching $M$ of $C-v$, and recolor all edges of $M$ red, and remove all edges of $(C-v)-M$ and the edge $v u_{2}$. Then we obtain a smaller fractional factor, a contradiction. Hence $C$ is a component of $F$.

It is easy to see that $C$ has a $\left\{P_{2}, P_{3}\right\}$-factor $F_{C}$. We recolor all edges of $P_{2}$-components and $P_{3}$-components of $F_{C}$ red, and remove all other edges of $C$. Then we obtain a smaller fractional factor, a contradiction. Therefore $F$ has no cycles, and the claim is proved.

Claim 2. Let $x$ and $y$ be two vertices of degrees at least 3 in $F$. Then $x$ and $y$ are not adjacent in $F$. If $x$ and $y$ are connected by a path whose inner vertices all have degree 2 in $F$, then the length of the path is 2 , and $\operatorname{deg}^{h}(x)=\operatorname{deg}^{h}(y)=k+\frac{1}{2}$ and the two edges in the path are blue edges.

Proof. If $x$ and $y$ are adjacent in $F$, then $F-x y$ is a smaller fractional factor, a contradiction. Assume that $x$ and $y$ are connected by a path $\left(x, u_{1}, u_{2}, \ldots, u_{n}, y\right)$ with $\operatorname{deg}_{F}\left(u_{i}\right)=2$ $(1 \leqslant i \leqslant n)$. If $n \geqslant 2$, then by removing $u_{n} y$ and recoloring $u_{n-1} u_{n}$ red and all the remaining edges of the path blue, the resulting subgraph is a smaller fractional factor, a contradiction. Therefore $n=1$, and the path is $\left(x, u_{1}, y\right)$.

If $\operatorname{deg}^{h}(x)<k+\frac{1}{2}$, then by removing $u_{1} y$ and recoloring $x u_{1}$ red, we obtain a smaller fractional factor, a contradiction. Hence $\operatorname{deg}^{h}(x)=\operatorname{deg}^{h}(y)=k+\frac{1}{2}$ by the symmetry. If $x u_{1}$ is red, then removing $u_{1} y$ we obtain a smaller fractional factor, a contradiction. Therefore $x u_{1}$ and $y u_{1}$ are blue edges, and the claim holds.

Claim 3. A leaf $z$ in $F$ is either contained in a star component, or adjacent to a vertex $x$ with $\operatorname{deg}_{F}(x) \geqslant 3$ and $\operatorname{deg}^{h}(x)=k+\frac{1}{2}$. 
Proof. Let $z$ be a leaf of $F$, and $D$ be the component of $F$ containing $z$. Assume that $D$ is a path $\left(z, u_{1}, u_{2}, \ldots, u_{n}, y\right)$ such that $\operatorname{deg}_{F}\left(u_{i}\right)=2(1 \leqslant i \leqslant n)$ and $\operatorname{deg}_{F}(y)=1$. If $n \geqslant 2$, then by removing $u_{1} u_{2}$ and recoloring $u_{2} u_{3}$ red, we obtain a smaller fractional factor, a contradiction. If $n=1$, then $D=P_{3}=K_{1,2}$, which is a star.

Next assume that $F$ contains a path $\left(z, u_{1}, u_{2}, \ldots, u_{n}, x\right)$ such that $\operatorname{deg}_{F}\left(u_{i}\right)=2$ $(1 \leqslant i \leqslant n)$ and $x$ has degree at least 3 in $F$. If $n \geqslant 2$, then by removing $u_{n} x$, and recoloring $u_{n-1} u_{n}$ and $z u_{1}$ red, and recoloring all other remaining edges (if any) of the path blue, we obtain a smaller fractional factor, a contradiction. If $n=1$, then $F-u_{1} x$ is a smaller fractional $\left[1, k+\frac{1}{2}\right]$-factor, a contradiction. Therefore $z$ and $x$ are adjacent.

Moreover, if $D$ contains exactly one vertex $x$ of degree at least 3 , by the same argument given above, we see that every leaf of $D$ is adjacent to $x$ and thus $D$ is a star; otherwise, $D$ contains another vertex $y$ of degree at least 3 , then by Claim $2, \operatorname{deg}^{h}(x)=k+\frac{1}{2}$. Thus the claim is proved.

Claim 4. (i) If uv is an edge such that $\operatorname{deg}_{F}(u) \geqslant 3$ and $\operatorname{deg}_{F}(v)=2$, then $\operatorname{deg}^{h}(u)=k+\frac{1}{2}$ and uv is a blue edge. (ii) If $x y$ is a red edge, then one of $x$ and $y$ is a leaf of $F$.

Proof. Let $u v$ be an edge of $F$ such that $\operatorname{deg}_{F}(u) \geqslant 3, \operatorname{deg}_{F}(v)=2$ and $\operatorname{deg}^{h}(u)<k+\frac{1}{2}$. Let $z_{1}$ be a vertex adjacent to $v$. If $\operatorname{deg}_{F}\left(z_{1}\right) \geqslant 3$, then by Claim 2 , we have $\operatorname{deg}^{h}(u)=k+\frac{1}{2}$, a contradiction. If $\operatorname{deg}_{F}\left(z_{1}\right)=1$, then it contradicts Claim 3. Hence $\operatorname{deg}_{F}\left(z_{1}\right)=2$. By removing $u v$ and recoloring $v z_{1}$ red, we obtain a smaller fractional factor, a contradiction. Hence if $u v$ is an edge with $\operatorname{deg}_{F}(u) \geqslant 3$ and $\operatorname{deg}_{F}(v)=2$, then $\operatorname{deg}^{h}(u)=k+\frac{1}{2}$. It is sufficient to prove (ii) to deduce that $u v$ is a blue edge.

Assume to the contrary that $x y$ is a red edge and neither $x$ nor $y$ is a leaf. If $\operatorname{deg}_{F}(x) \geqslant$ 3 and $\operatorname{deg}_{F}(y) \geqslant 3$, then $F-x y$ is a smaller fractional factor, a contradiction. Hence, by symmetry, we may assume that $\operatorname{deg}_{F}(x)=2$ and $\operatorname{deg}_{F}(y) \geqslant 3$. Let $z_{1}$ be another vertex adjacent to $x$. If $\operatorname{deg}_{F}\left(z_{1}\right) \geqslant 3$, then $F-x z_{1}$ is a smaller fractional factor since $x y$ is a red edge, a contradiction. By Claim $3, \operatorname{deg}_{F}\left(z_{1}\right)=2$. From Claim $2, F$ has no path connecting two vertices of degrees at least 3 and passing through $\left(z_{1}, x, y\right)$. Hence we may assume that there is a path $\left(z, u_{1}, \ldots, u_{n}=z_{1}, x, y\right)$ with $\operatorname{deg}_{F}(z)=1, \operatorname{deg}_{F}\left(u_{i}\right)=2$ $(1 \leqslant i \leqslant n)$ and $\operatorname{deg}_{F}(x)=2$. By Claim $3, u_{1}=x$, which is a contradiction since $u_{n}=z_{1} \neq x$. Therefore Claim 4 holds.

Claim 5. Every component $D$ of $F$ is isomorphic to a graph in $\left\{K_{1,1}, K_{1,2}, \ldots, K_{1, k}\right.$, $\mathcal{T}(2 k+1)\}$.

Proof. If $D$ is a star with center $u$, then $\operatorname{deg}^{h}(u) \leqslant k+\frac{1}{2}$, which implies $\operatorname{deg}_{D}(u) \leqslant k$ since every pendant edge of $D$ is red. So we may assume that $D$ is not a star, which implies that $D$ has at least two vertices of degrees at least 3 by Claim 3 .

Let $v$ be a vertex of degree at least 3. If $x v$ is a red edge, then $x$ is a leaf and vice versa. If $v y$ is a blue edge, then $\operatorname{deg}_{F}(y)=2$ and vice versa. Consequently, $D$ is a tree in $\mathcal{T}(2 k+1)$.

With Claim 5, we complete the proof. 


\section{Acknowledgements}

The research was carried out during the first and the third authors' visits to Xi'an Jiaotong University. The support and hospitality from the university were greatly appreciated. We are grateful to the anonymous referees for their constructive comments.

\section{References}

[1] J. Akiyama and M. Kano. Factors and factorizations of graphs. Lecture Notes in Mathematics (LNM 2031), Springer, 2011.

[2] A. Amahashi and M. Kano. On factors with given components. Discrete Math., 42:1-6, 1982.

[3] R.P. Anstee. Simplified existence theorems for $(g, f)$-factors. Discrete Appl. Math., 27:29-38, 1990.

[4] K. Heinrich, P. Hell, D. Kirkpatrick and G. Liu. A simple existence ciriterion for $(g<f)$-factors. Discrete Math., 85:313-317, 1990.

[5] M. Kano, H. Lu and Q. Yu. Component factors with large components in graphs. Appl. Math. Lett., 23:385-389, 2010.

[6] M. Kano and A. Saito. Star-factors with large components. Discrete Math., 312:20052008, 2012.

[7] W.T. Tutte. The 1-factors of oriented graphs. Proc. Amer. Math. Soc., 4:922-931, 1953.

[8] J. Yang, Y. Ma and G. Liu. Fractional $(g, f)$-factors in graphs. Appl. Math. J. Chinese Univ. Ser. A, 16:385-390, 2001.

[9] Q. Yu and G. Liu. Graph factors and matching extensions. Springer, 2009.

[10] Y. Zhang, G. Yan, M. Kano. Star-like factors with large components. J. Oper. Res. Soc. China, 3:81-88, 2015. 